\title{
aPDT for periodontitis treatment in ovariectomized rats under systemic nicotine
}

\author{
Valdir Gouveia Garcia ${ }^{\mathrm{a}, *}$, Erivan Clementino Gualberto Júnior ${ }^{\mathrm{a}}$, Edilson Ervolino ${ }^{\mathrm{a}, \mathrm{b}}$, \\ Maria José Hitomi Nagata ${ }^{a}$, Juliano Milanezi de Almeida ${ }^{a}$, Letícia Helena Theodoro ${ }^{a}$ \\ ${ }^{a}$ São Paulo State University (UNESP), Group for the Research and Study of Laser in Dentistry, Department of Surgery and Integrated Clinic, Division of Periodontics, \\ School of Dentistry, Araçatuba, 1193, Jose Bonifácio, 16015-050, Araçatuba, SP, Brazil

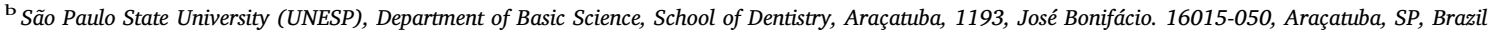

\section{A R T I C L E I N F O}

\section{Keywords:}

Nicotine

Ovariectomy

Periodontitis

Photochemotherapy

RANK ligand

\begin{abstract}
A B S T R A C T
Background: This study evaluated the antimicrobial photodynamic therapy (aPDT) as an adjunctive therapy to scaling and root planning (SRP) for the treatment of experimental periodontitis (EP) in ovariectomized rats under systemic nicotine.

Methods: Female ovariectomized rats $(\mathrm{n}=180)$ were divided into two groups: vehicle administration (Veh) and nicotine administration (Nic). Mini-pumps containing either vehicle or nicotine were inserted in the rats 30 days before the induction of EP, which was induced by placing a ligature around the left mandibular first molar. The rats were randomly divided into three treatment subgroups: SRP, SRP plus low-level laser therapy (LLLT), and SRP plus aPDT. aPDT consisted of the application of a phenothiazine photosensitizer followed by LLLT. Ten animals from each group were euthanized at days 7, 15, and 30 after periodontal treatment. The furcation region was evaluated using histological, histometric analyses and immunolabelling for PCNA, TRAP, RANKL, and OPG. Results: Nicotine administration resulted in greater bone loss (BL). aPDT resulted in lower BL compared to SRP. aPDT showed higher quantities of PCNA-positive cells compared to SRP, regardless of the nicotine status. aPDT resulted in less recruitment of osteoclasts and lower RANKL immunolabelling compared to LLLT and SRP. Conclusion: aPDT was effective in animals treated with nicotine.
\end{abstract}

\section{Introduction}

Periodontal disease (PD) is a dental plaque-induced inflammation of the periodontal tissues that results in altered bone metabolism and progressive damage to tooth support in susceptible individuals [1]. Individually, smoking and oestrogen deficiency are risk factors for PD [1] and disrupt the repair process after periodontal therapy [2-4]. However, the simultaneous effects of nicotine and oestrogen deficiency on the repair of periodontal tissue after periodontal treatment are unknown. Nicotine and ovariectomy interfere with bone metabolism by changing the RANK/RANKL/OPG system, leading to the downregulation of OPG and the upregulation of RANKL, which favours increased clastic cells and, consequently, the PD process [3-5].

Scaling and root planing (SRP) is the most common periodontal treatment, which has proven clinical effectiveness in terms of decreasing the probing pocket depth, reducing inflammation, and improving the clinical attachment level [6]. The limitations of SRP become more evident particularly if the disease has led to the formation of deep periodontal pockets and furcation-involved lesions [6,7].

Antimicrobial photodynamic therapy (aPDT) is an innovative method for treating periodontal infections. aPDT may be effective as an adjunctive therapy to periodontal mechanical debridement, especially in cases of advanced PD and in sites with difficult access [3-5]. The biological principle of aPDT is based on the reaction among photosensitizer or photoactive dye with a laser light source (low-power visible light with a suitable wavelength), and oxygen, which produces toxic forms of oxygen species that can lead to the inactivation of target cells, microorganisms, or molecules [8].

In this context, the aim of the present study was to evaluate the effect of aPDT as adjunctive therapy to the conventional mechanical treatment of SRP alone on local inflammatory response, periodontal repair, alveolar bone loss, osteoclast recruitment, and local bone metabolism regulators in ovariectomized rats with experimentally induced periodontitis under systemic nicotine.

\footnotetext{
* Corresponding author.

E-mail addresses: vgouveia@foa.unesp.br (V.G. Garcia), erivangualberto@ufam.edu.br (E.C. Gualberto), eervolino@foa.unesp.br (E. Ervolino), mjnagata@uol.com.br (M.J.H. Nagata), jumilanezi@hotmail.com (J.M. de Almeida), letheodoro@uol.com.br (L.H. Theodoro).
} 


\section{Materials and methods}

This study was conducted on 180 female adult Wistar rats, all 3 months of age at the start. The oestrous cycle was monitored prior to the surgical procedures, and rats exhibiting abnormal cycles were removed. The remaining rats $(n=180)$ were ovariectomized $(\mathrm{OVX})$ and randomly distributed into two groups: group Veh $(n=90)$ for vehicle administration (solution of $0.9 \%$ sodium chloride) and group Nic $(\mathrm{n}=90)$ for nicotine administration. For all surgical procedures, the rats were anesthetized with ketamine $(70 \mathrm{mg} / \mathrm{kg}$ ) and xylazine $(6 \mathrm{mg}$ / $\mathrm{kg}$ ) administered via intramuscular injection. All protocols described herein were approved by the Institutional Review Board of São Paulo State University, Araçatuba, SP, Brazil (no.2010/005074).

\subsection{Oestrous cycle and radioimmunoassay}

Intact female rats were studied after at least one complete oestrous cycle. To confirm the success of ovariectomy surgery, each rat was monitored 2 weeks after osmotic pump placement and 1 week after dental treatment. Changes in vaginal smears during days $4-5$ of the oestrous cycle were observed for all rats.

A radioimmunoassay was performed to verify the oestrogen levels and to confirm the ovariectomy. The radioimmunoassay followed the protocol described by Gualberto et al. [5]. The lowest detectable concentration in this assay was $2.8087 \mathrm{pg} / \mathrm{mL}$, and the intrassay coefficients of variation were $6.29 \%$ (High control) and $0.04 \%$ (Low control).

\subsection{Protocol for the administration of vehicle or nicotine}

Thirty days before ligature placement, the rats were anesthetized, and osmotic mini-pumps (Alzet model \#2006, Durect Corp., Palo Alto, CA, USA) containing nicotine or vehicle were surgically inserted subcutaneously in the backs of the rats. This administration followed the protocol described by Gualberto et al. [5].

\subsection{Ovariectomy surgery}

Bilateral OVX was performed in 180 rats from a dorsal approach 21 days before the induction of experimental periodontitis (EP) [5]. The ovary and surrounding fat were excised. Each animal received an intramuscular injection of 24,000 IU of penicillin (Pentabiótico* Veterinário Pequeno Porte, Animal Health Fort Dodger Ltd., Campinas, SP, Brazil) in the immediate post-operative period.

\subsection{Experimental periodontitis protocol}

Thirty days after the mini-pumps were inserted, one left mandibular first molar from each rat in both groups was selected to receive a cotton ligature in a submarginal position to induce EP. Ligatures were removed from all rats after 7 days. Rats in each group (Veh and Nic) were randomly assigned through a computer-generated table to one of three treatment subgroups: SRP, only SRP; SRP plus low level laser therapy (LLLT) or SRP plus aPDT. Each subgroup had 30 rats. All treatment procedures were performed by the same experienced operator (ECGJ) [5].

\subsection{SRP treatment}

SRP was performed with manual curettes (\#1-2 Mini Five Gracey curettes, Hu-Friedy, Chicago, IL, USA) through 10 distal-mesial traction movements in both the buccal and lingual aspects. The furcation and interproximal regions of each tooth were scaled with the same curettes through cervical-occlusal traction movements [5].

\subsection{LLLT treatment}

The laser used in this study was an indium-gallium-aluminiumphosphorous laser (TheraLase, DMC Equipment, São Carlos, SP, Brazil) with a wavelength of $660 \mathrm{~nm}$ and a spot size of $0.0283 \mathrm{~cm}^{2}$. The laser was applied to the buccal and lingual aspects of the left mandibular first molar perpendicularly and in contact with the gingiva. The therapeutic laser was activated at a power of $0.035 \mathrm{~W}$ for $12 \mathrm{~s}$ for each buccal and lingual surface ( $24 \mathrm{~s}$ per tooth), with $0.14 \mathrm{~J}$ per point of energy and $14.82 \mathrm{~J} / \mathrm{cm}^{2}$ per surface of energy density. Each tooth received a total energy density of $29.64 \mathrm{~J} / \mathrm{cm}^{2}$ [5].

\section{7. aPDT treatment}

The left mandibular first molar was submitted to SRP and the application of $1 \mathrm{~mL}$ of 7-amino-8-methyl-phenotiazin-3-ylidene-dimethylammonium (phenothiazine; Sigma Chemical Co., St Louis, MO, USA) solution $(100 \mu \mathrm{g} / \mathrm{mL})$ followed by the application of the laser. The phenothiazine was slowly dropped into the periodontal pocket using a syringe and needle $(13 \times 0.45 \mathrm{~mm})$ without a bevel. After $1 \mathrm{~min}$ of phenothiazine application, LLLT, using the same parameters as described above, was applied to each buccal and lingual aspect perpendicularly and in contact with the gingivae [5].

\subsection{Experimental periods}

Ten rats from each treatment subgroup were euthanized at days 7 , 15 , and 30 after the periodontal treatment by the administration of a lethal dose of thiopental $(150 \mathrm{mg} / \mathrm{kg}$; Cristália, Itapira, SP, Brazil). Their jaws were removed and fixed with $4 \%$ formaldehyde in $0.1 \mathrm{M}$ phosphate buffer ( $\mathrm{pH} \mathrm{7.4)} \mathrm{for} 48 \mathrm{~h}$.

\subsection{Laboratory procedures}

The specimens were demineralized in a solution of $10 \%$ EDTA, subjected to conventional histological processing, and embedded in paraffin. Some sections were stained with haematoxylin and eosin (HE) or Masson trichrome (MT). Other sections were submitted to the indirect immunoperoxidase method with the following primary antibodies: anti-PCNA (VP 980 - Vector Laboratories, Burlingame, CA, USA), anti-TRAP, anti-RANKL, and anti-OPG (Goat anti-TRAP - SC 30833, Goat anti-RANKL - SC 7628, Goat anti-OPG - SC 8468, Santa Cruz Biotechnology, Santa Cruz, CA, USA). The histological and immunohistochemical processes followed the protocol described by Gualberto et al. [5].

\subsection{Histopathological analysis}

The sections stained with HE were analysed by a certified histologist blinded to the treatments (EE). The following parameters were examined: nature and extension of the inflammatory process; presence of necrotic tissue; presence, extent, and nature of resorption of the bone, cementum, and dentin; state of the vasculature; structuring pattern of the extracellular matrix of the periodontal tissues; and cellularity pattern of the periodontal tissues. The entire furcation region of the left mandibular first molar was analysed [5].

\subsection{Histometric analysis}

The sections stained with TM were used to assess the alveolar bone loss (BL) in the furcation region. One trained examiner (EE), who was blinded to the groups and treatments, selected the sections for histometric analysis. Another calibrated examiner (ECGJ), also blinded to groups and treatments, conducted the histometric analysis. The histometric analysis followed the protocol described by Gualberto et al. [5]. 


\subsection{Immunohistochemical analysis}

The examiner who was blinded to groups and treatments (EE) conducted the immunohistochemical analyses. The values for each section were measured three times by the same examiner on different days to reduce variations in the data. Immunolabelling for RANKL and OPG was analysed in the entire furcation region of the mandibular first molar under $400 \times$ magnification. A semi-quantitative analysis was performed using three histological sections for each animal. PCNA- and TRAP-positive cells were analysed in the central area of the inter-radicular septum of an area of $1000 \times 1000 \mu \mathrm{m}$ under $200 \times$ magnification. Quantitative analysis was performed for TRAP and PCNA using five sections from each animal. The criteria for immunolabelling followed those adopted by Gualberto et al. [5].

\subsection{Intra-examiner reproducibility}

Before the histometric and immunohistochemical analyses were performed, the examiner was trained and then calibrated by performing double measurements of 30 specimens with a one-week interval. Pearson's correlation coefficient revealed a very high correlation (0.95) between the two sets of measurements for both the histometric and immunohistochemical analyses.

\subsection{Statistical analysis}

The results demonstrated that with a sample size of $10(p<0.05)$, the power of the study would be $95 \%$. The hypothesis that neither alveolar BL nor the number of TRAP- and PCNA-positive cells in the furcation region would differ among groups and time points was tested using software (BioEstat 5.3, Manaus, AM, Brazil). The normality of the histometric data was analysed using the Shapiro-Wilk test. The intraand inter-group analyses were performed with analysis of variance (ANOVA) $(p<0.05)$. When ANOVA detected a significant difference, multiple comparisons were performed using Tukey's test $(p<0.05)$ for BL and TRAP-positive cells. Bonferroni's test $(p<0.05)$ was used for PCNA-positive cells.

\section{Results}

\subsection{Hormone radioimmunoassay}

At euthanasia time the mean concentration of $17 \beta$-estradiol in the serum was $22.10 \mathrm{pg} / \mathrm{mL}$ to group Veh and $20.78 \mathrm{pg} / \mathrm{mL}$ to group Nic. These concentrations demonstrate the oestrogen deficiency of the rats in this study.

\subsection{Histological analyses}

The most relevant results of this analysis were found at 7 days after local treatment. At 15 days, there was a proportional decrease of the inflammatory infiltrate in all groups, and at 30 days there was no difference between the groups.

\subsubsection{SRP treatment}

At 7 and 15 days, both groups treated with SRP showed a connective tissue composed of a delicate network of collagen fibres, a moderate quantity of fibroblasts, and an intense inflammatory infiltrate composed predominantly of polymorphonuclear neutrophils, which was exacerbated to group Nic (Fig. 1A, D, G, J). The interradicular septum showed thin trabecular bone, and the outer contour was quite irregular due to the large amount of osteoclasts with weak activity, mainly due to Nic.

\subsubsection{LLLT treatment}

At 7 days, the Veh group and Nic group treated with LLLT had connective tissue composed of a more delicate network of collagen fibres, a moderate amount of fibroblasts, and moderate inflammatory infiltrate in Veh, which was more intense in Nic (Fig. 1B, E, H, K). At 15 days, the histological characteristics were similar to those described in the previous period however there was a proportional decrease in the volume occupied by the inflammatory infiltrate.

\subsection{3. aPDT treatment}

At 7 days after aPDT treatment, both groups showed a connective tissue with a moderate amount of collagen fibres, rich in fibroblasts and mild inflammatory infiltrate (Fig. 1C, F, I, L). At 15 days, the volume of the inflammatory infiltrate suffered a proportional reduction in both groups. The outline of the interradicular septum was quite irregular, especially in group Nic, which exhibited strong osteoclast activity. The interradicular septum comprised finer trabecular bone at 7 days and became thicker after 15 days.

\subsection{Histometric assessment}

Animals treated with aPDT showed less BL when compared to animals treated with SRP in group Veh at 7 and 30 days and group Nic at 7 and 15 days. The animals treated with LLLT showed less BL compared to animals treated with the SRP in group Veh at 7 days (Fig. 2).

\subsection{Immunohistochemical assessment}

The immunohistochemical technique for detecting PCNA, TRAP, RANKL, and OPG showed high specificity, which was confirmed by the total absence of labelling in the negative control. The immunolabelling was predominantly expressed in connective cells in the bone surface for PCNA, osteoclasts for TRAP, and osteoblasts and fibroblasts for RANKL and OPG.

\subsubsection{Immunohistochemical assessment for PCNA}

In both groups (Veh and Nic), animals treated with aPDT showed a greater number of PCNA-positive cells at 7 days when compared to SRP $(p<0.05)$. The animals treated with LLLT in group Nic at 15 days had higher numbers of PCNA-positive cells when compared to SRP $(p<0.05)$. At 15 days, group Nic showed a higher number of PCNApositive cells compared group Veh $(p<0.05)$, except for the animals of group Nic treated with SRP (Fig. 3A-G).

\subsubsection{Immunohistochemical assessment for TRAP}

The animals of group Nic showed a greater number of TRAP-positive cells after local therapy when compared to group Veh animals in all experimental periods $(p<0.05)$. Animals treated with aPDT showed a lower number of TRAP-positive cells when compared to SRP-treated animals on day 7 in the group Veh and in all time points in group Nic $(p<0.05)$. The animals treated with LLLT showed a lower number of TRAP-positive cells when compared to animals treated with SRP at 7 days in both groups $(p<0.05)$ (Fig. 4A-G).

\subsubsection{Immunohistochemical assessment for RANKL}

The pattern of immunolabelling for RANKL was moderate at 7 days post-treatment and decreased gradually at 15 and 30 days post-treatment in group Veh in all treatments, especially aPDT. Group Nic showed a high pattern of immunolabelling in all experimental periods in animals treated with LLLT and SRP. In animals treated with aPDT, the pattern of immunolabelling underwent a gradual reduction (Fig. 5A-F).

\subsubsection{Immunohistochemical assessment for $O P G$}

The pattern of immunolabelling for OPG was low at 7 and 15 days post-treatment, while suffering a slight increase at day 30 in all groups treated with LLLT and SRP. In animals treated with aPDT, immunolabelling was consistently low at 7 days but increased at 15 and 30 days in all experimental groups (Fig. 5G-L). 

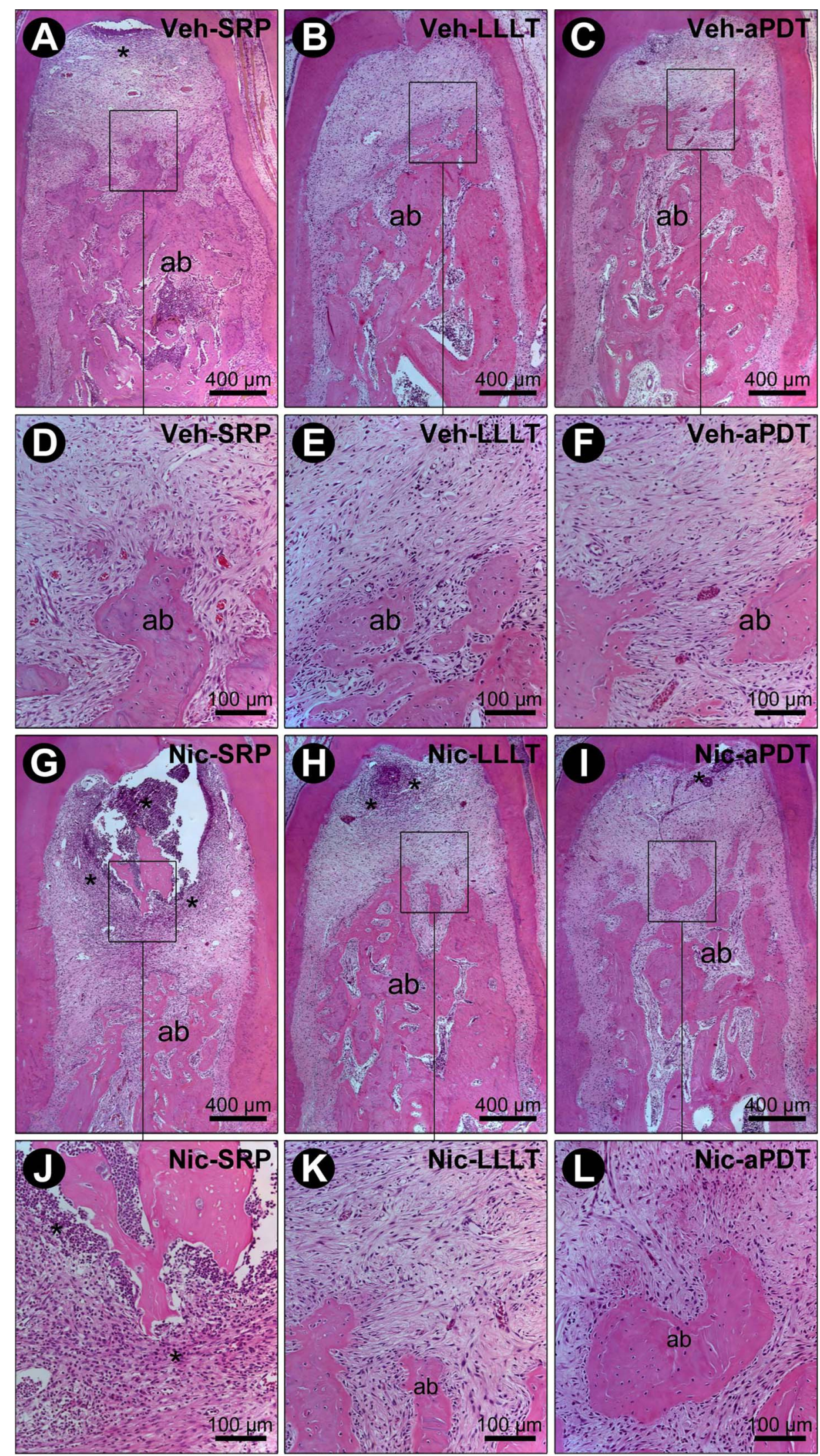

Fig. 1. Photomicrographs showing the histological appearance of periodontal tissues 7 days after SRP alone or in combination with LLLT or aPDT in the different experimental groups. A-F: histopathological characteristics of the furcation region of the left mandibular first molar in Veh (A-F) at 7 days after treatment with SRP (A, D), LLLT (B, E), and aPDT (C, F). G-L: histopathological characteristics of the furcation region of the left mandibular first molar in Nic (G-L) at 7 days after treatment with SRP (G, J), LLLT (H, K), and aPDT (I, L). Abbreviations and symbols: ab, alveolar bone; asterisks, inflammatory infiltrate. Staining: haematoxylin and eosin (HE). Scale bars: A-C and G-I, $400 \mu \mathrm{m}$; D-F and J-L, $100 \mu \mathrm{m}$. Original Magnification: A-C and G-I, x40; D-F and J-L, x200.

\section{Discussion}

This study evaluated the effect of aPDT as adjuvant to SRP in the treatment of EP in ovariectomized rats under systemic nicotine. The prevalence and severity of PD are higher in smokers than in non-smokers [1]. In addition, treatment of PD is less effective in smokers compared to non-smokers [9]. Postmenopausal women become more susceptible to $\mathrm{PD}$, and this problem is due in part to oestrogen 


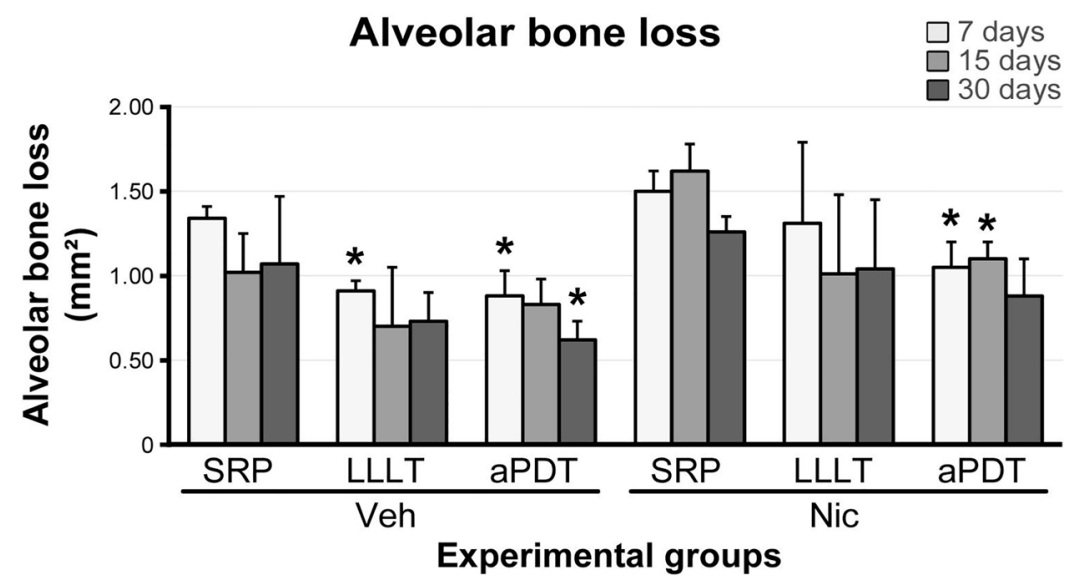

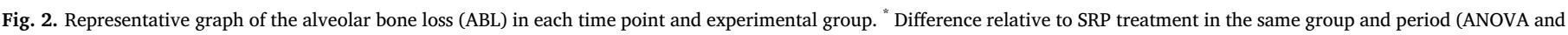
Tukey's test, $p<0.05$ ).

deficiency, which results in BL and inflammation [2] and can also be considered a risk factor for PD [1]. Additionally, cigarette smoke has been associated with several bone disturbances, like osteoporosis and periodontal BL. These conditions may be attributed partly to the known inflammatory effects of tobacco smoke, although a direct effect on bone cells may also occur [10].

Nicotine and ovariectomy interfere with bone metabolism through the RANK/RANKL/OPG system by favouring the increased clastic cells and delaying the process of periodontal healing after SRP [3-5]. In this study, the process of periodontal healing after treatment with SRP was substantially impaired by nicotine; however, aPDT, as adjuvant to SRP, minimized the deleterious effects of nicotine to regulate the magnitude of the local inflammatory response and thus modulate the RANK/ RANKL/OPG system.

A previous study [3] from our group showed that oestrogen deficiency accelerated bone loss and delayed the repair process after periodontal treatment in ovariectomized rats with EP when compared to group Sham. Oestrogen deficiency alters bone metabolism by increasing the magnitude of the inflammatory response and by regulating the RANK/RANKL/OPG system by increasing the levels of RANKL and thus inducing osteoclastogenesis and stimulating osteoclast activation $[3,11]$.

In this study, more aggressive insult to the periodontium was promoted via the combined effect of oestrogen deficiency and nicotine, which acted simultaneously on the periodontal repair process after local treatment in rats with EP. The results showed that nicotine did not increase BL under oestrogen deficiency; however, there was a tendency for animals treated with nicotine to show higher BL. A possible explanation for this result may be the fact that, in this study, the nicotine did not negatively affect the circulating oestrogen levels. However, in a previous study [5], we found that nicotine accelerated bone loss in induced periodontitis in intact female rats with normal circulating oestrogen levels. These results may suggest that compared to nicotine, oestrogen deficiency plays a more active role in the progression of the $\mathrm{BL}$ and that nicotine's deleterious effects are most noticeable in normal circulating oestrogen levels.

Additionally, histological analyses showed that groups treated with nicotine exhibited necrotic bone spicules surrounded by inflammatory cells in the furcation, and the interradicular septum comprised fine bone trabeculae with high numbers of osteoclasts. In this study, oestrogen deficiency and nicotine exhibited delayed periodontal repair processes after the completion of local treatments. However, the animals treated with aPDT showed less BL compared to those treated with LLLT and SRP in both groups. Furthermore, aPDT showed lesser inflammatory infiltrate and interradicular septum with more regular contour and bone trabeculae, which gradually thickened over time.
LLLT-treated animals exhibited less BL when compared to SRP in the group Veh, but it did not show the same result in group Nic. The best result of the aPDT may be due to its antimicrobial and bio-stimulator effect and its performance in regions unreached by SRP.

The host inflammatory response to smoking and periodontopathogens is considered a major causative factor in the local tissue destruction observed in periodontitis $[12,13]$. The association of smoking status with periodontal destruction can thus be correlated with the increased mRNA expression of IL-1 $\beta$ [14]. Nicotine induces the production of IL- $1 \beta$ and IL- 8 via the $\alpha 7 \mathrm{nAChR/NF- \kappa B}$ pathway in human periodontal ligament cells [15], and through this pathway, it induces osteoclastogenesis and osteoclast activation [13]. In addition to this indirect effect, nicotine has the ability to act directly on osteoclast precursors, inducing its osteoclastogenic differentiation [10]. The stimulatory behaviour appears to be dependent on the stage of osteoclastic differentiation of the precursor cells, which means, in the absence of macrophage colony-stimulating factor (M-CSF) and RANKL, it only favours the initial stages of osteoclast differentiation, while in the presence of the growth factors, a significant increase in their resorbing ability is also achieved [10].

In this study, nicotine affected the RANK/RANKL/OPG axis, resulting in upregulated RANKL and downregulated OPG and a higher number of TRAP-positive cells, while aPDT led to the downregulation of RANKL and the upregulation to OPG and a lower number of TRAPpositive cells. The animals treated with LLLT also showed a lower number of TRAP-positive cells in both groups. These results show the potential effect of aPDT and LLLT on the regulation of bone metabolism in systemically adverse situations. Recently, Theodoro et al. [16,17] found similar results in the treatment of periodontitis induced in rats subjected to 5 -fluorouracil chemotherapy. The RANKL/RANK/OPG axis is significantly involved in osteoclast activation and differentiation and bone remodelling $[4,12]$.

The effect of aPDT on the RANK/RANKL/OPG axis may be due to its antimicrobial and biostimulatory effect. When it promotes bacterial killing, aPDT inactivates virulence factors and host inflammatory cytokine, modulating the exacerbated immune-inflammatory response initiated by the action of lipopolysaccharide [18]. In addition, aPDT acts on the cellular immune response by stimulating the action of macrophages [19], and the biostimulating effect of LLLT acts on the immune system by enhancing leukocyte phagocytosis and chemotaxis [20]. However, there is still no consensus on the effects of aPDT and laser on the inflammatory mediators. A recent systematic review has concluded that it remains controversial whether aPDT or LLLT as adjuvants are effective in reducing gingival crevicular fluid inflammatory mediators in periodontal disease because of non-standard laser parameters and short-term follow-up. These conflicting findings show the 

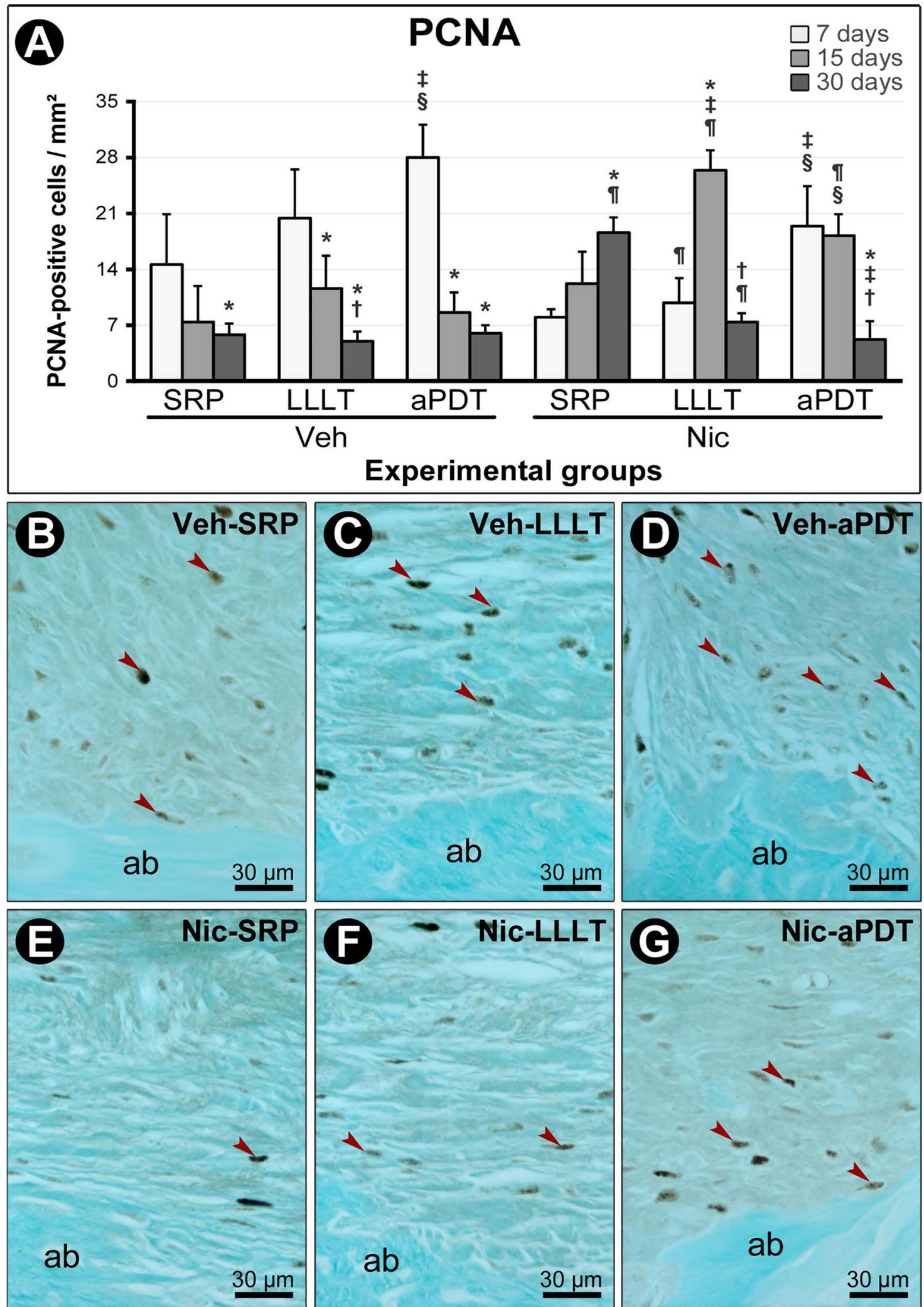

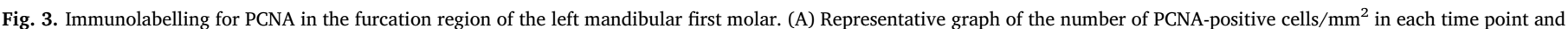

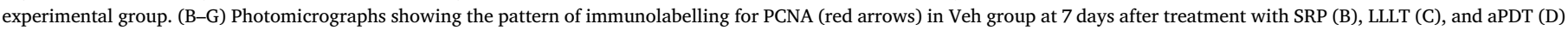

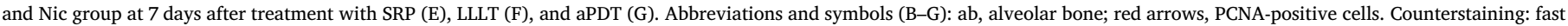

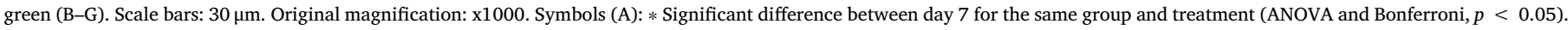

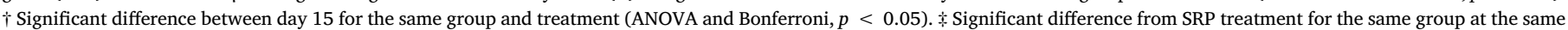

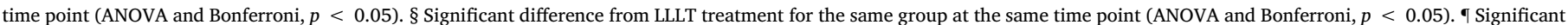
difference from group Veh for the same treatment and time point. (ANOVA and Bonferroni, $p<0.05$ ). 


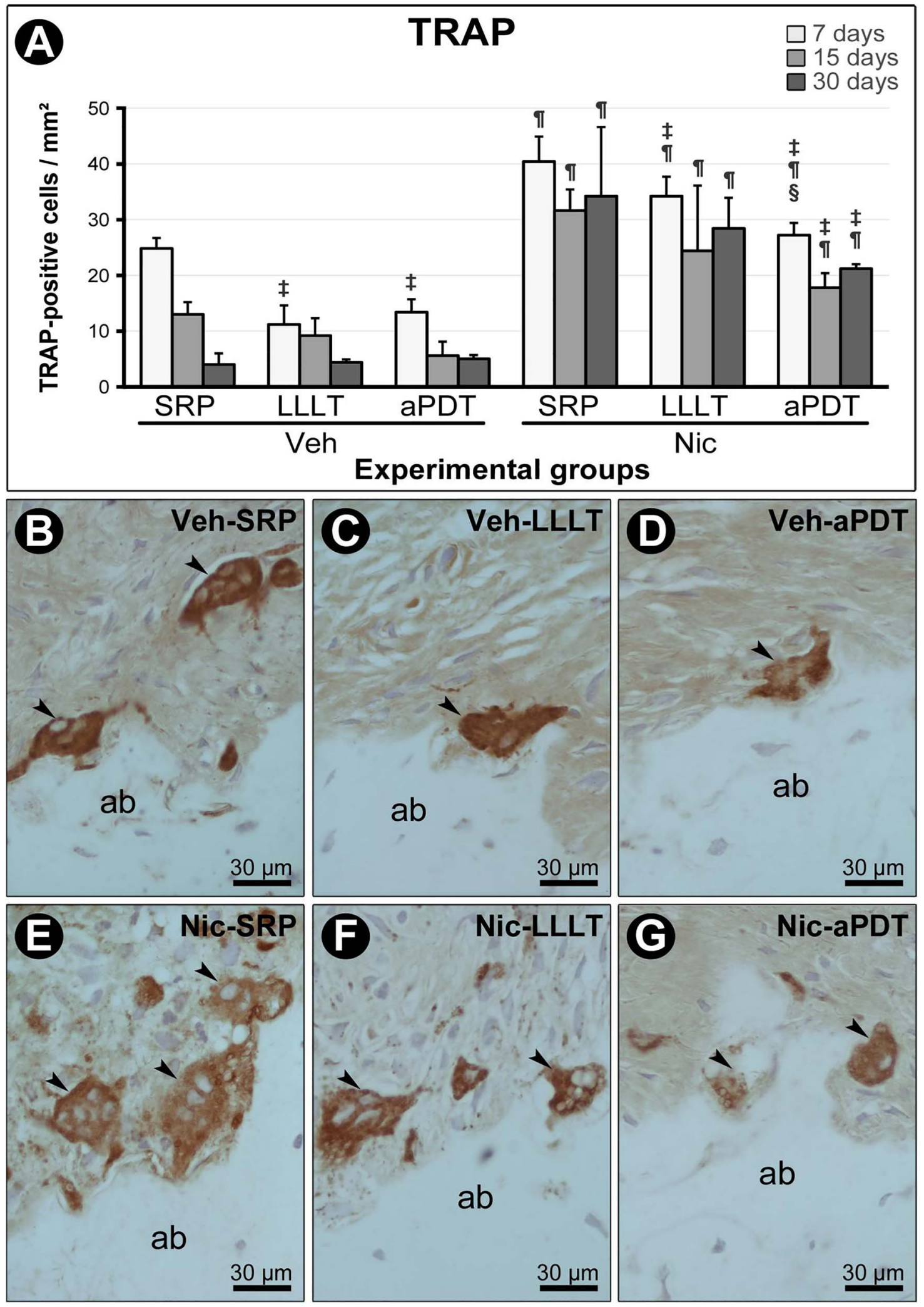

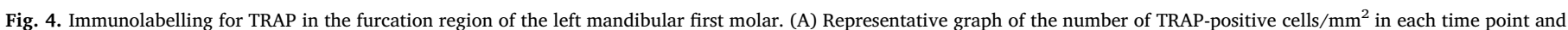

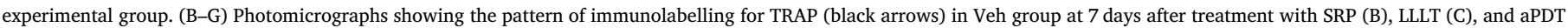

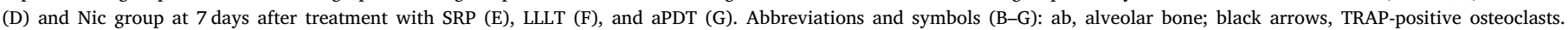

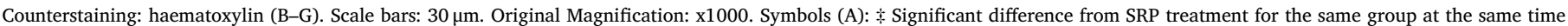

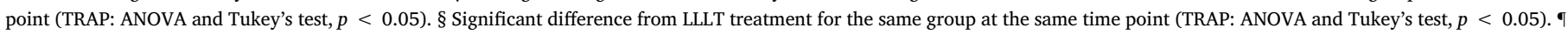
Significant difference from group Veh for the same treatment and time point. (TRAP: ANOVA and Tukey's test, $p<0.05$ ). 

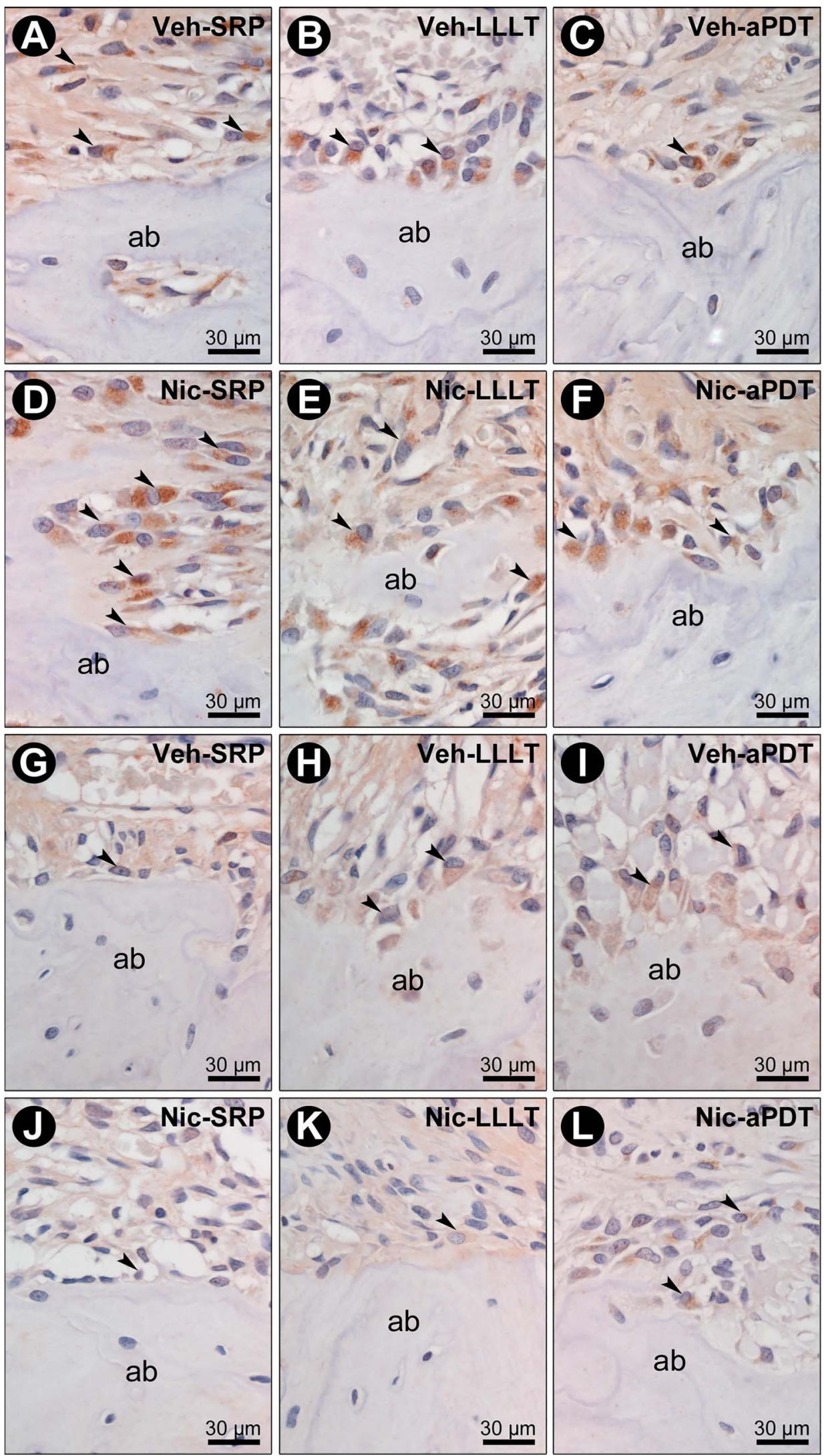

Fig. 5. Immunolabelling for RANKL and OPG in the furcation region of the left mandibular first molar. (A-F) Photomicrographs showing the pattern of immunolabelling for RANKL (arrows) in Veh group at 7 days after treatment with SRP (A), LLLT (B), and aPDT (C) and Nic group at 7 days after treatment with SRP (D), LLLT (E), and aPDT (F). (G-L) Photomicrographs showing the pattern of immunolabelling for OPG (arrows) in Veh group at 30 days after treatment with SRP (G), LLLT (H), and aPDT (I) and Nic group at 30 days after treatment with SRP (J), LLLT (K), and aPDT (L). Abbreviations and symbols: ab, alveolar bone; arrows, immunolabelling cell. Counterstaining: haematoxylin. Scale bars: $30 \mu \mathrm{m}$. Original Magnification: x1000. need for additional studies for a better understanding of the subject [21].

Evidence shows divergent results with the use of aPDT in many fields of study. One of the reasons for this divergence may be in the protocol used to perform this therapy, such as the light source used and the number of sessions. Our research group [3-5,17,22] has achieved promising results with aPDT as adjunct to SRP by performing a singlesession and using laser light with the parameters described above. In 
this aspect, the results found in the present study can contribute to the consolidation of the aPDT protocol proposed here. Another study in our group did not demonstrate a significant reduction of BL in animals treated with multiple aPDT sessions when compared to a single session of this therapy [22]. This divergence of protocols also occurs with LLLT. Studies in humans have found promising results with a single LLLT session adjunct to SRP [23,24]; in turn Pjecic et al. [25] found promising results with 10 LLLT sessions.

The results showed that nicotine inhibited the cell proliferation and delayed the repair. In this study, Nicotine conducted a resorptive activity, demonstrated by immunolabelling for TRAP, which hampered the periodontal repair. The nicotine-exerted toxic effects on M-CSF are likely related, at least in part, to the altered expression of multiple proteins essential to the health and proliferation of these cells [26]. However, here aPDT minimized this deleterious effect of nicotine. Animals treated with aPDT showed a higher number of PCNA-positive cells compared to SRP and LLLT at 7 days. Moreover, the same group showed fewer TRAP-positive cells in all experimental periods. This fact justifies the improvement in the tissue repair process provided by the aPDT.

\section{Conclusion}

Within the limits of this study, we concluded that aPDT favored the periodontal repair process by inhibiting the bone resorption and stimulating cell proliferation in ovariectomized rats with induced periodontal disease regardless the systemic influence of nicotine. LLLT was effective in controlling bone loss and resorptive activity in ovariectomized rats without systemic nicotine. Therefore, aPDT can be considered a promising adjuvant for the treatment of periodontitis in oestrogen deficiency and under systemic nicotine.

\section{Conflict of interest}

The authors declare that they have no conflicts of interest. Valdir Gouveia Garcia declares that he has no conflicts of interest. Erivan Clementino Gualberto Júnior declares that he has no conflicts of interest. Edilson Ervolino declares that he has no conflicts of interest. Maria José Hitomi Nagata declares that she has no conflicts of interest. Juliano Milanezi de Almeida declares that he has no conflicts of interest. Letícia Helena Theodoro declares that she has no conflicts of interest.

\section{Research involving animals - ethical approval}

All applicable international, national, and/or institutional guidelines for the care and use of animals were followed. All procedures performed in studies involving animals were in accordance with the ethical standards of the institution or practice at which the studies were conducted.

\section{Acknowledgements}

This study was financially supported by the São Paulo Research Foundation-FAPESP, São Paulo, SP, Brazil (FAPESP process no.2011/ 00936-0 and no. 2010/15094-2) and Prointer (Unesp Process no. 2015/ 1028). The authors are grateful to Dr. Guilherme de Paula Nogueira (Faculty of Veterinary Medicine, UNESP, Araçatuba, SP, Brazil) for his guidance in performing radioimmunoassays.

\section{References}

[1] R.J. Genco, W.S. Borgnakke, Risk factors for periodontal disease, Periodontology 62 (2013) (2000) 59-94.
[2] N. An, O.O. Andrukhov, Y. Tang, F. Falkensammer, H.P. Bantleon, X. Ouyang, X. Rausch-Fan, Effect of nicotine and porphyromonas gingivalis lipopolysaccharide on endothelial cells in vitro, PLoS One 9 (2014) e96942.

[3] V.G. Garcia, E.C. Gualberto Júnior, L.A. Fernandes, A.F. Bosco, M.J.H. Nagata, C.A. Casatti, E. Ervolino, L.H. Theodoro, Adjunctive antimicrobial photodynamic treatment of experimentally induced periodontitis in rats with ovariectomy, $\mathrm{J}$ Periodontol. 84 (2013) 556-565.

[4] V.G. Garcia, L.A. Fernandes, V.C. Macarini, J.M. de Almeida, T.M. Martins, A.F. Bosco, M.J.H. Nagata, J.A. Cirelli, L.H. Theodoro, Treatment of experimental periodontal disease with antimicrobial photodynamic therapy in nicotine-modified rats, J. Clin. Periodontol. 38 (2011) 1106-1114.

[5] E.C. Gualberto Jr., L.H. Theodoro, M. Longo, V.C. Novaes, M.J.H. Nagata, E. Ervolino, V.G. Garcia, Antimicrobial photodynamic therapy minimizes the deleterious effect of nicotine in female rats with induced periodontitis, Lasers Med. Sci. 31 (2016) 83-94.

[6] A.D. Haffajee, M.A. Cugini, S. Dibart, C. Smith, R.L. Kent Jr., S.S. Socransky, The effect of SRP on the clinical and microbiological parameters of periodontal diseases, J. Clin. Periodontol. 24 (1997) 324-334.

[7] H.C. Fleischer, J.T. Mellonig, W.K. Brayer, J.L. Gray, J.D. Barnett, Scaling and root planing efficacy in multirooted teeth, J. Periodontol. 60 (1989) 402-409.

[8] L.J. Tavares, L.J. Pavarina, A.C. Vergani, C.E.E.D. de Avil, The impact of antimicrobial photodynamic therapy on peri-implant disease: what mechanism are involved in this novel treatment? Photodiagn. Photodyn. Ther. (2017), http://dx. doi.org/10.1016/j.pdpdt.2016.11.016 (Epub 2016 Dec 6).

[9] P.M. Preshaw, L. Heasman, F. Stacey, N. Steen, G.I. McCracken, P.A. Heasman, The effect of quitting smoking on chronic periodontitis, J. Clin. Periodontol. 32 (2005) 869-879.

[10] J. Costa-Rodrigues, I. Rocha, M.H. Fernandes, Complex osteoclastogenic inductive effects of nicotine over hydroxyapatite, J. Cell. Physiol. (2017), http://dx.doi.org/ $10.1002 / \mathrm{jcp} .25956$.

[11] X. Yan, T.W. Ye, Early molecular responses of bone to estrogen deficiency induced by ovariectomy in rats, Int. J. Clin. Exp. Med. 8 (2015) 5470-5477.

[12] D.L. Cochran, Inflammation and bone loss in periodontal disease, J. Periodontol. 79 (8 Suppl) (2008) 1569-1576.

[13] T. Breivik, Y. Gundersen, P. Gjermo, S. von Hörsten, P.K. Opstad, Nicotinic acethylcholine receptor activation mediates nicotine induced enhancement of experi mental periodontitis, J. Periodontal Res. 44 (2009) 110-116.

[14] A. Meenawat, V. Govila, S. Goel, S. Verma, K. Punn, V. Srivastava, R.S.Dolas Evaluation of the effect of nicotine and metabolites on the periodontal status and the mRNA expression of interleukin- $1 \beta$ in smokers with chronic periodontitis, $J$. Indian Soc. Periodontol. 19 (2015) 381-387.

[15] L. Wu, Y. Zhou, Z. Zhou, Y. Liu, Y. Bai, X. Xing, X. Wang, Nicotine induces the production of IL-1 $\beta$ and IL- 8 via the $\alpha 7 \mathrm{nAChR} / \mathrm{NF}-\mathrm{kB}$ pathway in human periodontalligament cells: an in vitro study, Cell Physiol. Biochem. 34 (2014) 423-431.

[16] L.H. Theodoro, M. Longo, E. Ervolino, C. Duque, M.L. Ferro-Alves, N.Z. Assem, L.M. Louzada, V.G. Garcia, Effect of low-level laser therapy as an adjuvant in the treatment of periodontitis induced in rats subjected to 5-fluorouracil chemotherapy, J. Periodontal. Res. 51 (2016) 669-680.

[17] L.H. Theodoro, M. Longo, V.C.N. Novaes, D.M. Janjacomo Miessi, M.L. Ferro-Alves, E. Ervolino, J.M. de Almeida, V.G. Garcia, Low-level laser and antimicrobial photodynamic therapy on experimental periodontitis in rats submitted to chemotherapy by 5-fluorouracil, Support. Care Cancer (2017), http://dx.doi.org/10 1007/s00520-017-3738-0.

[18] P. Braham, C. Herron, C. Street, R. Darveau, Antimicrobial photodynamic therapy may promote periodontal healing through multiple mechanisms, J. Periodontol. 80 (2009) 1790-1798.

[19] S. Song, F. Zhou, W.R. Chen, D. Xing, PDT-induced HSP70 externalization up-regulates NO production via TLR2 signal pathway in macrophages, FEBS Lett. 587 (2013) 128-135.

[20] T.T. Tadakuma, Possible application of the laser in immunobiology, Keio. J. Med. 42 (1993) 180-182.

[21] Z. Akrama, T. Abduljabbar, S. Sauro, U. Daood, Effect of photodynamic therapy and laser alone as adjunct to scaling and root planing on gingival crevicular fluid inflammatory proteins in periodontal disease: a systematic review, Photodiagn. Photodyn. Ther. 16 (2016) 142-153.

[22] V.G. Garcia, M. Longo, L.A. Fernandes, E.C. Gualberto Jr., C.S. Santinoni, A.F. Bosco, M.J. Nagata, L.H. Theodoro, Treatment of experimental periodontitis in rats using repeated adjunctive antimicrobial photodynamic therapy, Lasers Med. Sci. 28 (2013) 143-150.

[23] S. Malgikar, S.H. Reddy, S.V. Sagar, D. Satyanarayana, G.V. Reddy, J.J. Josephin, Clinical effects of photodynamic and low-level laser therapies as an adjunct to scaling and root planing of chronic periodontitis: a split-mouth randomized controlled clinical trial, Indian J. Dent. Res. 27 (2016) 121-126.

[24] N.T. Nguyen, M.R. Byarlay, R.A. Reinhardt, D.B. Marx, T.A. Meinberg, W.B. Kaldahl, Adjunctive non-surgical therapy of inflamed periodontal pockets during maintenance therapy using diode laser: a randomized clinical trial, J. Periodontol. 86 (2015) 1133-1140.

[25] A. Pjecic, D. Kojovic, L. Kesic, R. Obradovic, The effects of low level laser irradiation on gingival inflammation, Photomed. Laser Surg. 28 (2010) 69-74.

[26] H.L. Zeng, Y.L. Qin, H.Z. Chen, Q.Q. Bu, Y. Li, Q. Zhong, X.A. Han, J. Chen, P.X. Yu, G.X. Liu, Effects of nicotine on proliferation and survival in human umbilical cord mesenchymal stem cells, J. Biochem. Mol. Toxicol. 28 (2014) 181-189. 